\title{
$R E V 3 L$ modulates cisplatin sensitivity of non-small cell lung cancer H1299 cells
}

\author{
WENJIE WANG $^{1-3}$, WENJIONG SHENG ${ }^{1,2}$, CHENXIAO YU $^{1,2}$, JIANPING CAO $^{1,2}$, \\ JUNDONG ZHOU $^{4}$, JINCHANG WU ${ }^{4}$, HUOJUN ZHANG ${ }^{5}$ and SHUYU ZHANG ${ }^{1,2}$
}

\author{
${ }^{1}$ School of Radiation Medicine and Protection, and Jiangsu Provincial Key Laboratory of Radiation Medicine \\ and Protection, Medical College of Soochow University; ${ }^{2}$ Collaborative Innovation Center of Radiation Medicine \\ of Jiangsu Higher Education Institutions and School for Radiological and Interdisciplinary Sciences (RAD-X), \\ Soochow University; ${ }^{3}$ Cyrus Tang Hematology Center, Soochow University, Suzhou $215123 ;{ }^{4}$ Department of Radio- \\ Oncology, Nanjing Medical University Affiliated Suzhou Hospital, Suzhou 215001; ${ }^{5}$ Department of Radiation \\ Oncology, Shanghai Changhai Hospital, the Second Military Medical University, Shanghai 200433, P.R. China
}

Received March 17, 2015; Accepted June 3, 2015

DOI: 10.3892/or.2015.4121

\begin{abstract}
Lung cancer remains the leading cause of cancerrelated mortality worldwide and non-small cell lung cancer (NSCLC) accounts for approximately $80-85 \%$ of all cases of lung cancer. Cisplatin plays a significant role in the management of human lung cancer. Translesion DNA synthesis (TLS) is involved in DNA damage repair. DNA polymerase $\zeta$ (Pol $\zeta$ ) is able to mediate the DNA replication bypass of DNA damage, which is suggested to be involved in chemoresistance. REV3L is the catalytic subunit of Pol $\zeta$. Due to its critical role in translesion DNA synthesis, whether REV3L modulates cisplatin response in NSCLC cells remains unknown. In this study, REV3L overexpression and silencing H1299 cell lines were established. The reports showed that cisplatin induced the expression of REV3L by recruiting Sp1 to its promoter. Similar results were obtained when the ability of the cells to express luciferase from a platinated plasmid was measured. Co-transfection of the reporter with the REV3L overexpression vector or REV3L plus REV7L significantly enhanced the reporter activity. Nuclear condensation and fragmentation of shRNA-REV3L H1299 cells were more pronounced than shRNA-NC H1299 cells after cisplatin exposure, indicating that REV3L overexpression abolished cisplatin-induced DNA
\end{abstract}

Correspondence to: Dr Huojun Zhang, Department of Radiation Oncology, Shanghai Changhai Hospital, the Second Military Medical University, No. 168 Changhai Road, Shanghai 200433, P.R. China E-mail: chyyzhj@163.com

Professor Shuyu Zhang, School of Radiation Medicine and Protection, Medical College of Soochow University, No. 199 Renai Road, Suzhou 215123, P.R. China

E-mail: zhang.shuyu@hotmail.com

Key words: non-small cell lung cancer, REV3L, cisplatin, chemosensitivity damage. Moreover, a forced expression of REV3L conferred the resistance of H1299 cells to cisplatin, whereas the knockdown of REV3L sensitized cisplatin efficacy in H1299 cells. Taken together, we demonstrated that inhibition of REV3L sensitized lung cancer H1299 cells to cisplatin treatment. Thus, REV3L may be a novel target for the chemotherapy of NSCLC.

\section{Introduction}

Lung cancer remains the leading cause of cancer-related mortality worldwide and non-small cell lung cancer (NSCLC) accounts for approximately $80-85 \%$ of all cases of lung cancer (1). The overall prognosis and survival rate of patients with advanced NSCLC remain unsatisfactory, with a median survival time of 8-11 months and a one-year survival rate of $30 \%(2,3)$. Chemotherapy plays a significant role in the management of human lung cancer $(4,5)$. Cisplatin (DDP) is widely used to treat many types of solid tumors and cisplatinbased adjuvant chemotherapy has significantly improved the progression-free survival of cancer patients, which has translated to a $5-10 \%$ improvement in the cure rate $(6,7)$. However, few patients experience complete responses to chemotherapy, mainly because of the resistance of tumor cells and/or tolerance of the surrounding normal tissues (8). Thus, novel approaches to enhance the cisplatin efficacy and reverse chemoresistance are required, as well as chemosensitizers to reduce the dose of drugs administered and the length of time resulting in reduced side effects.

Cisplatin induces DNA crosslinks and can induce cell apoptosis, as is the case with many other chemotherapeutic drugs (9). Cancer cells elicits DNA repair mechanisms including nucleotide excision repair (NER), base excision repair (BER), double-strand break repair (DSBR) and mismatch repair (MMR) (10-12). In addition, eukaryotic cells have a DNA post-replication repair system that is composed of translesion DNA synthesis (TLS) and homologous DNA recombination (HR) pathways. The ubiquitous TLS consists 
of a series of specialized polymerases, including polymerase (Pol) $\kappa, \zeta, \eta$ and $\iota$ (13). The catalytic REV3L subunit interacts with structural REV7L to form polymerase Pol $\zeta$. Pol $\zeta$ cannot add nucleotides across DNA lesions, but it can extend from primers with terminal mismatches, which makes Pol $\zeta$ crucial in translesion DNA synthesis (TLS). Pol $\zeta$ is able to mediate DNA replication bypassing DNA damage, which may prevent chromosome instability in cells and be considered as a suppressor of spontaneous tumorigenesis. However, Pol $\zeta$ lacks 3' to 5' exonuclease activity and can insert a nucleotide into the lesion to complete the bypass of the lesion, which is important for cell survival when confronted with DNA damage (14-16). Loss of REV3L (also known as REV3 in vetebrates) has shown increased sensitivity to exogenous insults. For example, REV3-null mouse-derived embryonic fibroblasts are more sensitive to UV- and $\gamma$-irradiation with increased chromosomal abnormalities (17). The above mentioned results indicated that REV $3 L$ is involved in cell tolerance to various types of DNA damage.

The REV3(L) protein was partially conserved from yeast to vertebrates. It has been previously reported that human Pol $\zeta$ is expressed in human cancers including lung, stomach and colorectal cancers $(18,19)$. Moreover, REV3L suppressed the focus formation suggesting a tumor-suppressor role. Due to its critical role in translesion DNA synthesis, whether REV3L is involved in chemoresistance remains unknown. In this study, we found that cisplatin induced the expression of REV3L by recruiting Sp1 to its promoter. Knockdown of REV3L sensitized cisplatin efficacy in H1299 cells.

\section{Materials and methods}

Reagents and plasmids. Benzo[a]pyrene $(\mathrm{B}[\mathrm{a}] \mathrm{P})$, nicotine and 4',6-diamidino-2-phenylindole dihydrochloride (DAPI) were purchased from Sigma-Aldrich (St. Louis, MO, USA). Cisplatin was purchased from Qilu Pharmaceutical Co., Ltd. (Shandong, China). Geneticin (G418) was obtained from Life Technologies (Gaithersburg, MD, USA).

REV3L overexpression and RNAi vectors. The pcDNA3.1/neo-REV3L plasmid was kindly provided by Dr Yoshiki Murakumo, Nagoya University Graduate School of Medicine, Nagoya, Japan. Four shRNAs targeting REV3L were designed and constructed by GenePharma (Shanghai, China). The information of the four shRNAs is provided in Table I.

Cell culture and transfection. The human H1299 lung cancer cell line was maintained in DMEM supplemented with $10 \%$ FBS and antibiotics (Gibco, Grand Island, NY, USA). The cells were grown in a $37^{\circ} \mathrm{C}$ incubator with $5 \% \mathrm{CO}_{2}$. The cells were transfected by Lipofectamine 2000 (Invitrogen Life Technologies, Carlsbad, CA, USA) with plasmids.

To generate stable $R E V 3 L$-overexpressing or -silencing clones, the cells were grown in a 24 -well culture plate to $70-80 \%$ confluence and then transfected with $1 \mu \mathrm{g}$ of pcDNA3.1, pcDNA-REV3L, shRNA-NC or shRNA targeting $R E V 3 L$ vector using Lipofectamine 2000 (Invitrogen Life Technologies) according to the manufacturer's instructions. The medium was replaced with DMEM containing $600 \mu \mathrm{g} / \mathrm{ml} \mathrm{G} 418$
Table I. Targeting sequences of shRNAs.

\begin{tabular}{lcc}
\hline Name & \multicolumn{1}{c}{ Targeting sequence } & $\begin{array}{c}\text { Location } \\
\text { (REV3L) }\end{array}$ \\
\hline shRNA-NC & 5'-GTCAATGGTCGTGTCGTGC-3' & \\
shRNA-1 & 5'-CGAAGATTGTGACCTGAATTA-3' & 3611-3632 \\
shRNA-2 & 5'-CTTCTGGTATGTCCTCAAAGA-3' & $4447-4468$ \\
shRNA-3 & 5'-AGGAAAGCCAAATGCCTAATA-3' & $4672-4693$ \\
shRNA-4 & 5'-CTCTAGTGATATCTCCAATTA-3' & $6859-6880$
\end{tabular}

$48 \mathrm{~h}$ post-transfection. After 3-4 weeks, G418-resistant colonies were selected and screened for $R E V 3 L$ expression by reverse transcriptase-PCR (RT-PCR). The REV3L-overexpressing and -silencing clones were cultured in DMEM supplemented with $10 \%$ fetal bovine serum in the presence of $300 \mu \mathrm{g} / \mathrm{ml} \mathrm{G} 418$ at $37^{\circ} \mathrm{C}$ in humidified air with $5 \% \mathrm{CO}_{2}$.

Reverse transcriptase-PCR analysis. Total RNA from lung tissues was extracted with TRIzol(InvitrogenLife Technologies) and reverse transcribed to cDNA using an oligo(dT $)_{12}$ primer and Superscript II (Invitrogen Life Technologies). The mRNA levels of target genes and the internal standard glyceraldehyde 3-phosphate dehydrogenase (GAPDH) were measured by RT-PCR or quantitative PCR (qPCR) in triplicate on a Prism 7500 real-time PCR machine (Applied Biosystems, Foster City, CA, USA). The specific primers for the genes are listed in Table II.

Immunostaining. H1299 cells were fixed with 4\% paraformaldehyde, washed with PBS, and permeabilized with $1 \%$ Triton X-100 in PBS. The cells were blocked with blocking buffer (PBS, 1\% Triton X-100, and 5\% BSA) and incubated at $4^{\circ} \mathrm{C}$ with the REV3L antibody (1:1000; Abnova, Taiwan) overnight. FITC-conjugated goat anti-mouse (1:100) was incubated for $30 \mathrm{~min}$ at room temperature. Nuclear counterstaining was performed using 4,6-diamidino-2-phenylindole (DAPI). Cells not treated with primary antibody served as the negative control.

Cell viability assay. Cell viability was evaluated using the 3-(4,5-dimethylthiazol-2-yl)-2,5-diphenyl-2H-tetrazolium bromide (MTT) assay. Cells were plated in 96-well plates. The following day, the cells were transfected with plasmids or shRNAs according to the experimental design. The cells were then incubated with $20 \mu \mathrm{l}$ MTT $(5 \mathrm{mg} / \mathrm{ml})$ for $4 \mathrm{~h}$. After the medium was removed, $100 \mu 1 \mathrm{DMSO}$ was added and the optical density (OD) at $490 \mathrm{~nm}$ was measured using a Microplate Reader (Bio-Rad, Hercules, CA, USA). The viability index was calculated as experimental OD value/control OD value. Three independent experiments were performed in quadruplicate.

Western blot analysis. The cells were lysed in lysis buffer (Promega, Madison, WI, USA) and centrifuged at $4{ }^{\circ} \mathrm{C}$ for $10 \mathrm{~min}$. The supernatant was collected and subjected to western blotting. Protein $(50 \mu \mathrm{g})$ from each lysate was fractionated by $10 \%$ SDS-PAGE and transferred to polyvinylidene difluoride membranes (Millipore, Bedford, MA, USA). After 
Table II. Primer sequences for RT-PCR and ChIP analysis.

RT-PCR

\begin{tabular}{lcc}
\cline { 2 - 3 } Gene & Forward primer & Reverse primer \\
\hline GAPDH & & \\
REV3L & 5'-GAAGGTGAAGGTCGGAGTC-3' & 5'-GAAGATGGTGATGGGATTTC-3' \\
\hline & 5'-CGCGTCAGTTGGGACTTAAG-3' & 5'-ACTATCGCCAACCTCAATGC-3' \\
\cline { 2 - 3 } Gene & Forward primer & ChIP \\
\hline Region 1 & Reverse primer \\
Region 2 & 5'-GAAGGTGAAGGTCGGAGTC-3' & 5'-GAAGATGGTGATGGGATTTC-3' \\
\hline
\end{tabular}

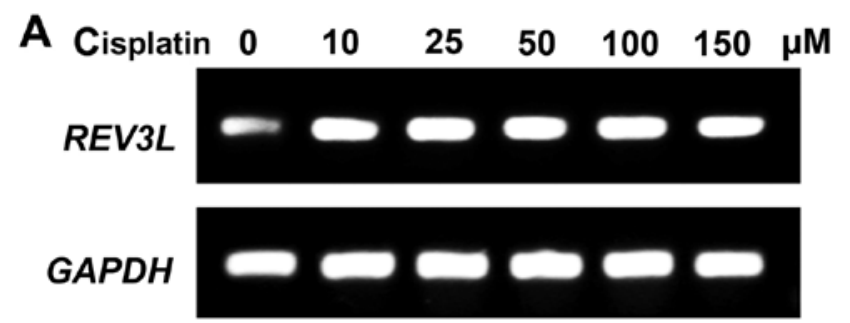

B

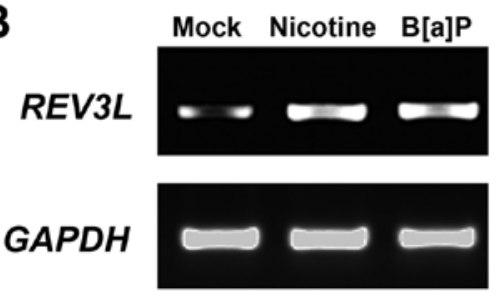

Figure 1. Cisplatin induces the expression of REV3L. (A) H1299 cells were exposed to various concentrations of cisplatin for $24 \mathrm{~h}$. (B) Cells were exposed to $\mathrm{B}[\mathrm{a}] \mathrm{P}$ or nicotine for $24 \mathrm{~h}$. After treatment, RNA was extracted by TRIzol and the expression of REV $3 L$ and the internal control GAPDH was detected by RT-PCR.

blocking with 5\% non-fat milk in PBS Tween-20 for $1 \mathrm{~h}$ at room temperature, the membranes were blotted with the appropriate Bax, Bcl 2 or GAPDH primary antibody (All from Santa Cruz Biotechnology, Inc., Santa Cruz, CA, USA) at a 1:1000 dilution. The membranes were then incubated with the appropriate horseradish peroxidase-conjugated secondary antibody at a 1:2000 dilution for $1 \mathrm{~h}$ at room temperature. After TBST washes, the blot was incubated in the ECL detection kit (Amersham Bioscience, Freiburg, Germany).

Plasmid reactivation assay. A plasmid reactivation assay was performed as previously reported (20). The pGL3-promoter mammalian expression vector (Promega) containing the Firefly luciferase gene expressed high-level Firefly luciferase in mammalian cells. The plasmid DNA was dissolved in buffer containing $10 \mathrm{mM}$ Tris and $1 \mathrm{mM}$ EDTA (pH 7.4) and incubated with different concentrations of cisplatin for $1 \mathrm{~h}$. The platinated DNA was then purified by ethanol precipitation and unbound free cisplatin was removed. This procedure resulted in plasmid DNA that was $>90 \%$ supercoiled as verified by gel electrophoresis. Similar levels of platination have previously been shown not to affect the efficiency of transfection (21). For the luciferase assay, the pGL3-Promoter reporter vector plus pRL-TK (Promega) was co-transfected with pcDNA3.1REV3L or shRNA-targeting REV3L. Luciferase activity was measured with the Dual-Luciferase Reporter Assay System (Promega). Promoter activities were expressed as the ratio of Firefly luciferase to Renilla luciferase activities.

Quantitative chromatin immunoprecipitation (ChIP). H1299 cells were used for ChIP assays. The EZ ChIP kit (Upstate Biotechnology, Inc, Lake Placid, NY, USA) was used according to the manufacturer's instructions as previously reported (22). Briefly, $5 \times 10^{6}$ cells were cross-linked with $1 \%$ formaldehyde and sonicated to $\sim 500$ bp fragments. ChIP was conducted with antibodies against PAX2 and IgG. Input control DNA or immunoprecipitated DNA was amplified in a $20-\mu 1$ reaction volume consisting of $4 \mu \mathrm{l}$ eluted DNA template and primers specific for REV3L promoter (Table II). The immunoprecipitated fragments and the inputs were amplified by RT-PCR and qPCR. Results for the immunoprecipitated fragments were calculated compared to the $\mathrm{Ct}$ values obtained for the input samples in each case and were expressed as a percentage of the input.

Measurement of apoptosis. Cells were transfected with shRNA-NC or shRNA-REV3L for $24 \mathrm{~h}$ prior to treatment with cisplatin. Apoptosis was measured using propidium iodide (PI)/ Annexin V double staining as per the manufacturer's instructions (Nanjing KeyGen Biotech. Co., Ltd., Nanjing, China). The cells were harvested $24 \mathrm{~h}$ after treatment with cisplatin and apoptotic fractions were measured using flow cytometry (Beckman Coulter, Miami, FL, USA). The Annexin $\mathrm{V}^{+} / \mathrm{PI}^{-}$ cells indicated early apoptosis, while the Annexin $\mathrm{V}^{+} / \mathrm{PI}^{+}$cells indicated late apoptosis. The percentage of the two types of cells was calculated.

Statistical analysis. Data were presented as the mean \pm standard error of the mean (SEM) of at least three independent experiments. Standard error bars were included for all the data points. The data were then analyzed using the Student's t-test when only two groups were present or assessed by one-way analysis of variance (ANOVA) when more than two groups were compared. Statistical analysis was performed using 

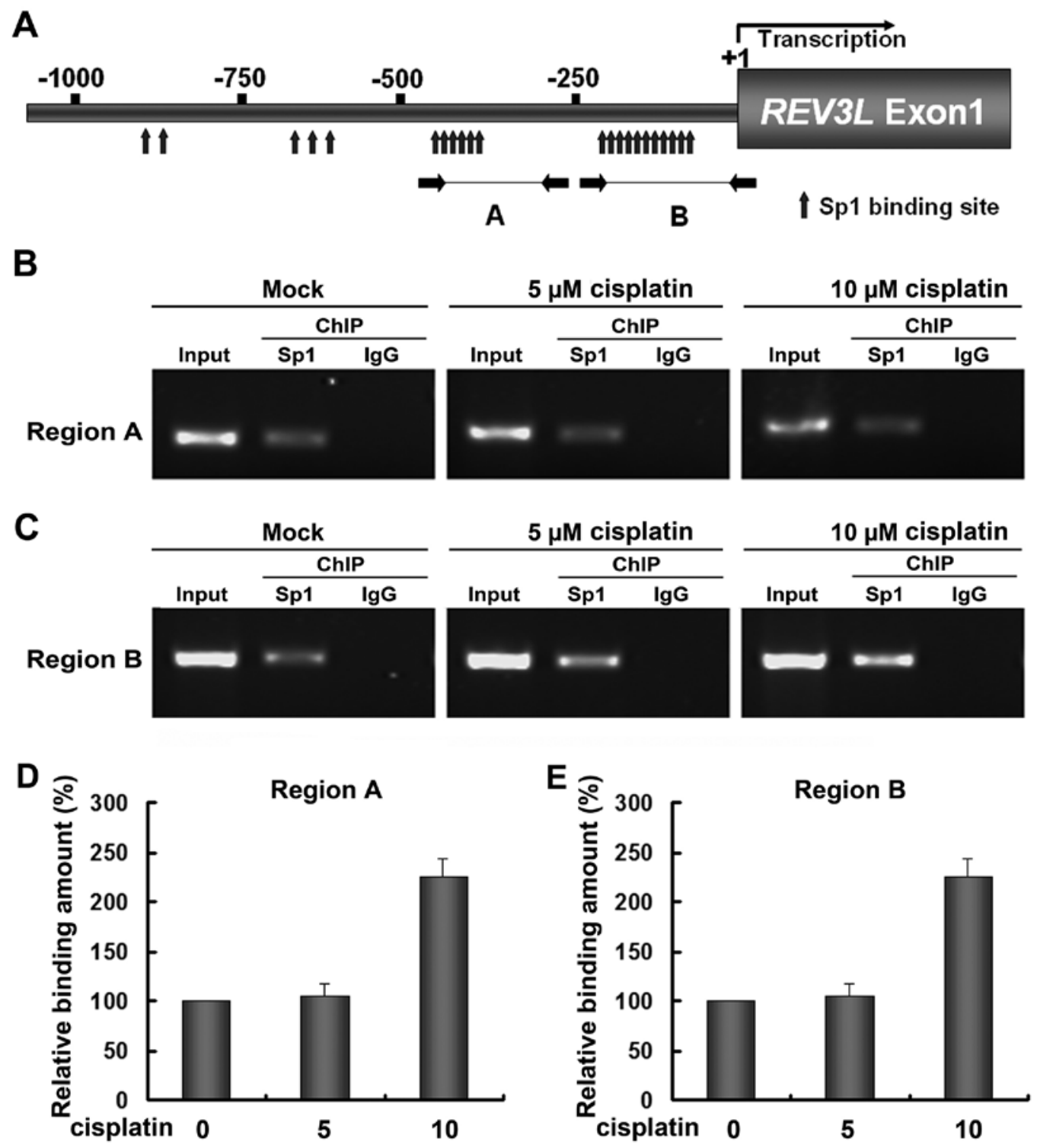

Figure 2. Sp1 is recruited for the cisplatin-induced REV3L expression. H1299 cells were mock-treated or treated with cisplatin for 24 h. (A) The H1299 cells were processed for ChIP using anti-Sp1 or IgG antibodies. (B) Relative binding amount of Sp1 to the REV $3 L$ promoter. The relative presence of Sp1 at the $R E V 3 L$ promoter was calculated relative to the corresponding input sample by real-time PCR.

SPSS software (Release 17.0, SPSS Inc.). Data were considered significant when $\mathrm{P}<0.05$.

\section{Results}

Cisplatin induces the expression of REV $3 L$. It has been suggested that cisplatin can induce an increase in REV3L mRNA levels in both normal and cancer cells. Thus, we investigated whether cisplatin also increases REV3L mRNA levels in human H1299 NSLSC cells. As shown in Fig. 1A, $10 \mu \mathrm{M}$ cisplatin increased the REV $3 L$ mRNA level while higher concentrations of cisplatin did not further increase REV3L expression. Moreover, DNA damage reagents $\mathrm{B}[\mathrm{a}] \mathrm{P}$ and nicotine upregulated REV3L expression in H1299 cells, indicating that REV3L was increased in response to DNA damage.

SPI is recruited in the REV3L promoter with cisplatin. Bioinformatic tools predicted that the promoter of REV3L harbors multiple binding sites of transcription factor Sp1 (Fig. 2A). Two dense Spl binding regions were observed, the upstream one located between -450 and -360 bp (Region A) and the downstream one located between -200 and $-75 \mathrm{bp}$ (Region B, relative to the transcription start site). To investigate whether the interaction between Sp1 and the REV $3 L$ promoter conferred the cisplatin-induced increase of REV3L, quantitative ChIP was performed. In H1299 cells, the REV3L promoter was specifically precipitated with a Spl antibody but not with the control IgG in the two Sp1 dense regions (Fig. 2B and $\mathrm{C}$ ), indicating the presence of Sp1 bound to the REV3L promoter in vivo. Results also showed that $5 \mu \mathrm{M}$ cisplatin did not change the relative binding amount of Sp1 to the REV3L promoter, whereas $10 \mu \mathrm{M}$ cisplatin significantly increased the amount of Sp1 bound to the REV3L promoter in the two regions (Fig. 3B-E). These results also indicated that changes in $R E V 3 L$ expression result in a corresponding change in $\mathrm{Sp} 1$ binding to the $R E V 3 L$ promoter. 
A

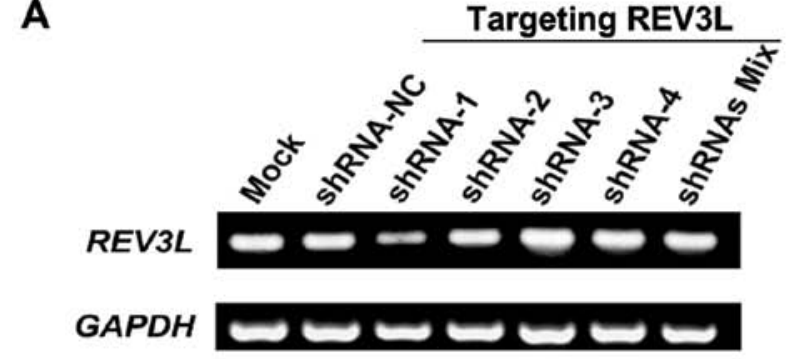

B

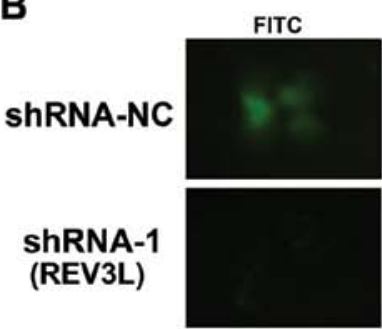

DAPI

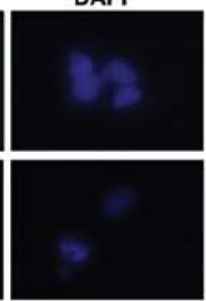

Merge

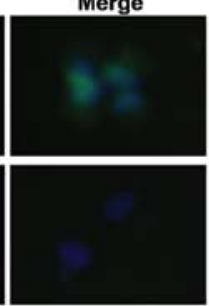

C

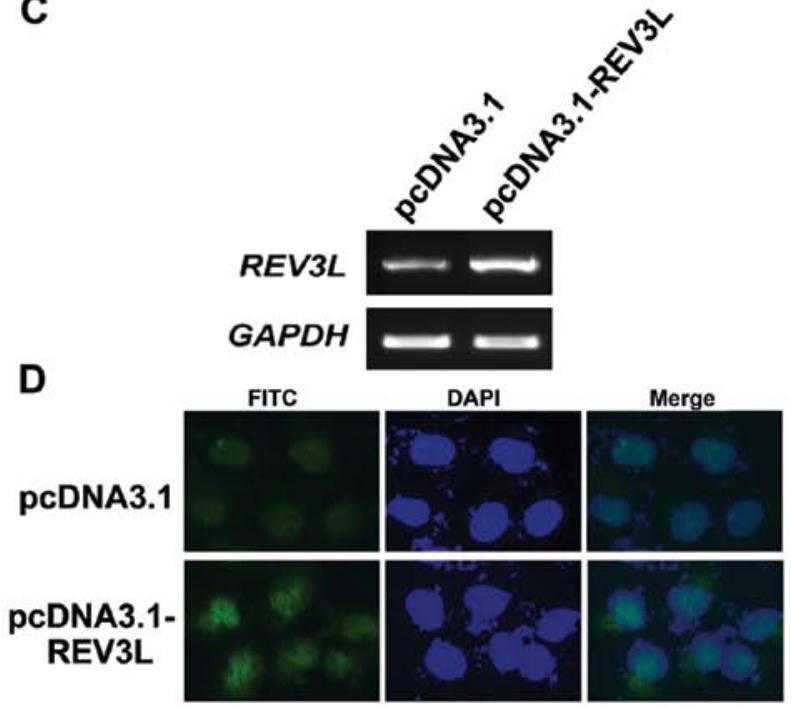

Figure 3. Evaluation of REV3L overexpression and silencing in H1299 cells. (A) H1299 cells were stably transfected with shRNA-NC or shRNA-targeting REV3L. REV3L mRNA expression was semi-quantified by RT-PCR. GAPDH was used as an internal control. (B) Stable H1299 cell lines were immunostained with an anti-REV3L and a FITC-conjugated secondary antibody. (C) H1299 cells were stably transfected with the REV3L overexpression vector. REV3L mRNA expression was semi-quantified by RT-PCR. GAPDH was used as an internal control. (D) Stable H1299 cell lines were immunostained with an anti-REV3L and a FITC-conjugated secondary antibody. Nuclei (blue) were stained with DAPI.
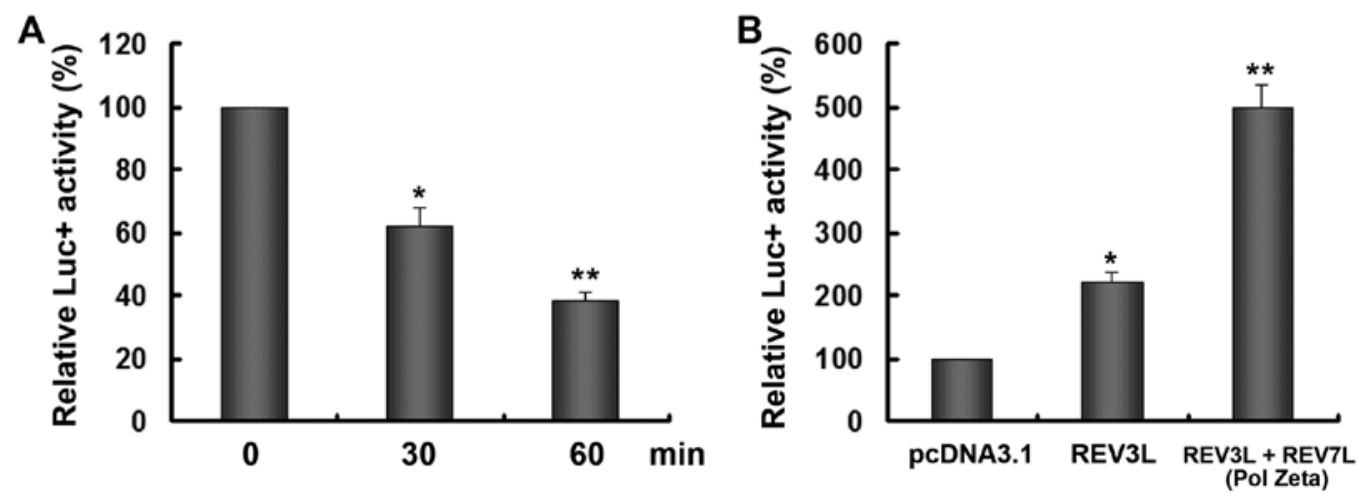

Figure 4. REV3L increases DNA repair following cisplatin treatment. (A) Luciferase assay of cells transfected with the cisplatin-treated pGL3-promoter reporter. ${ }^{*} \mathrm{P}<0.05$ and ${ }^{* *} \mathrm{P}<0.01$, compared with the mock-treated plasmid. (B) REV3L modulates the expression of cisplatin-treated reporter. The cisplatin-treated luciferase reporter was co-transfected with indicated vectors into H1299 cells. After 24 h of incubation, luciferase activity was measured as described in Materials and methods. ${ }^{*} \mathrm{P}<0.05 ;{ }^{* *} \mathrm{P}<0.01$, compared with pcDNA3.1-transfected cells.

Establishment of H1299 cell lines with overexpression and knockdown of REV3L. The function of REV $3 L$ in human lung cancer H1299 cells. We genetically manipulated $R E V 3 L$ expression in a cell line derived from H1299 was investigated. pcDNA3.1-REV3L was stably transfected into H1299 cells to generate REV3L-overexpressing cells. We also designed and constructed four shRNA vectors targeting different locations of the REV3L mRNA. REV3L cell lines stably transfected with the control, overexpression or shRNA vectors were screened by G418. As shown in Fig. 3A, compared with mock- or shRNANC-transfected H1299 cells, transfection of REV3L targeting shRNA-1 showed reduced REV $3 L$ transcripts (inhibition rate $71.4 \%$, relative to shRNA-NC-transfected cells). Other shRNAs did not show obvious silencing ability of REV $3 L$. To confirm this result, immunostaining was performed and the results demonstrated that in shRNA-1-transfected cells, the nuclear staining of REV3L was obviously reduced (Fig. 3B), validating the RT-PCR results. In addition, REV3L expression was increased in $R E V 3 L$-overexpressing stable cells, compared to the pcDNA3.1-transfected cells (Fig. 3C and D).

REV3L overexpression abolishes cisplatin-induced DNA damage. DNA is thought to be the primary biological target of cisplatin. The platinum atom of cisplatin forms covalent bonds with the N7 position of purine bases to form 1,2- or 1,3-intrastrand crosslinks and a lower percentage of interstrand crosslinks, both of which interfere with DNA transcription (23). To investigate whether REV3L affects cisplatin-treated DNA damage, the pGL3-promoter reporter was treated with cisplatin and then transfected into H1299 cells. The ability of cells in sweeping away cisplatin-induced adducts was assessed by determining the ability of the cell to successfully express the firefly luciferase from the extensively platinated reporter by treatment with cisplatin before transfection. As shown in Fig. 4A, the treatment of cisplatin decreased the reporter activity in a time-dependent manner possibly due 
A

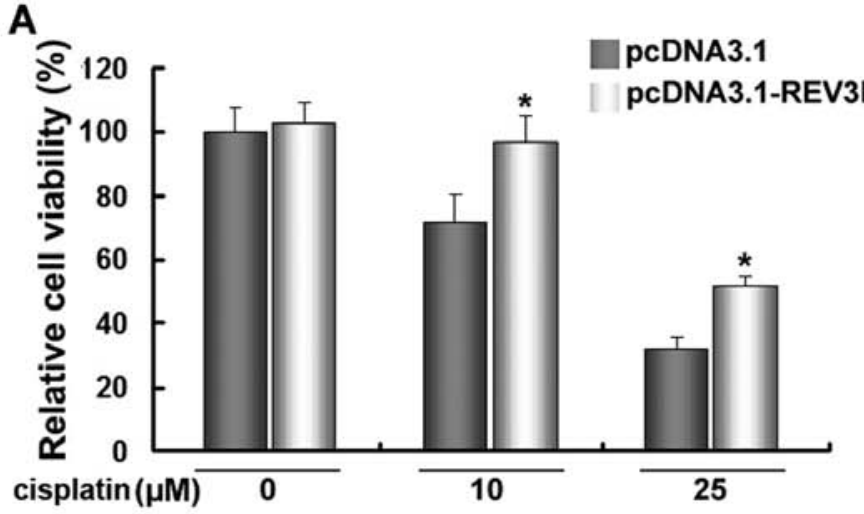

B

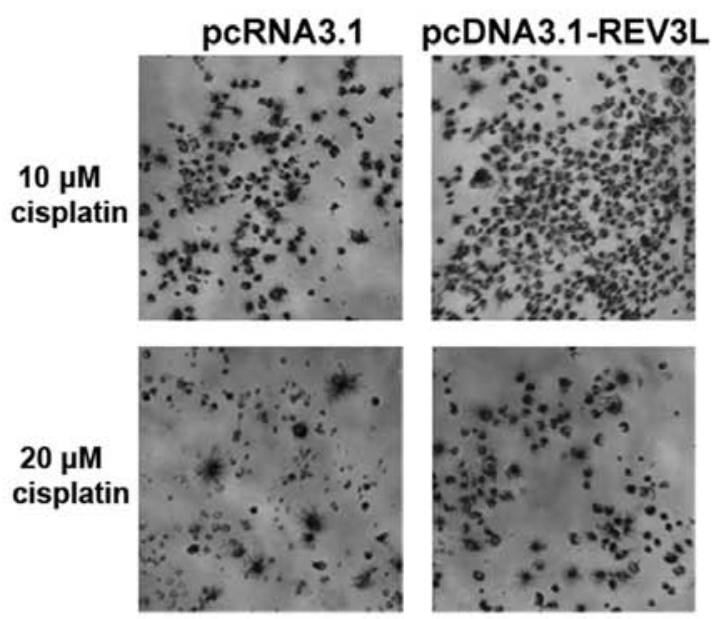

C

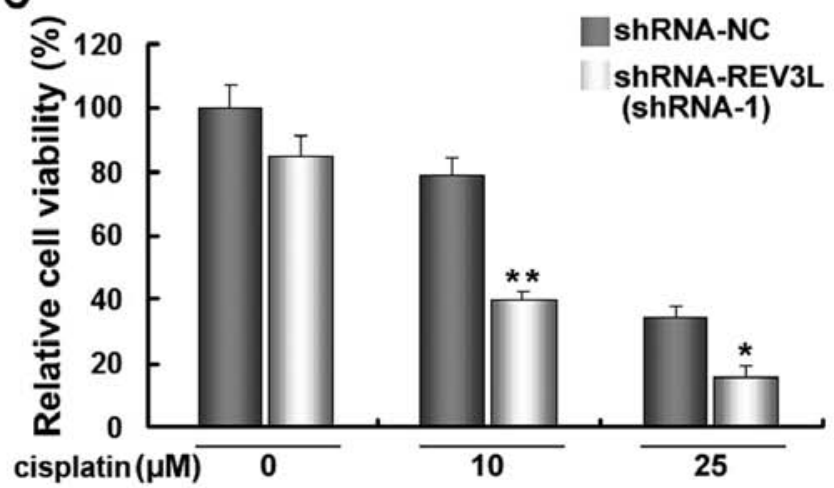

D
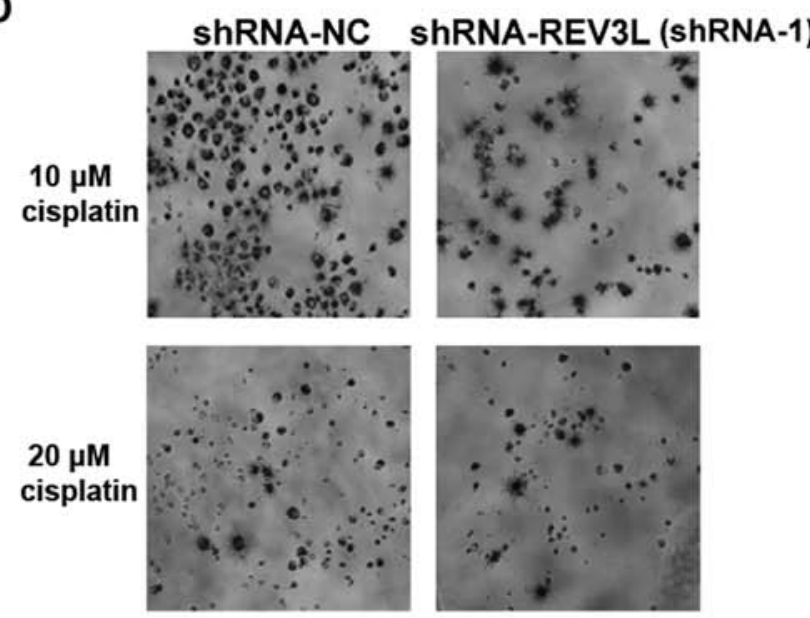

Figure 5. Knockdown of REV3L sensitizes cancer cells to cisplatin-induced cytotoxicity. (A) H1299 cells were stably transfected with pcDNA3.1 or pcDNA3.1-REV3L. The cells were treated with the indicated doses of cisplatin (DDP) for an additional $48 \mathrm{~h}$. Cell viability was determined by MTT. Data are presented as a percentage of viable cells, where vehicle-treated cells were regarded as $100 \%$ viable. Points show the mean \pm SEM of three independent experiments, ${ }^{*} \mathrm{P}<0.05$ and ${ }^{* *} \mathrm{P}<0.01$. (B) Representative MTT image of pcDNA3.1- or pcDNA3.1-REV3L-transfected H1299 cells treated with $10 \mu \mathrm{M}$ cisplatin for 48 h. (C) H1299 cells were stably transfected with shRNA-NC- or shRNA-targeting REV3L. The cells were treated with indicated doses of cisplatin for an additional 48 h. (D) Representative MTT image of shRNA-NC-transfected or REV3L silencing H1299 cells treated with 10 or $20 \mu \mathrm{M}$ cisplatin for 48 h.

to the crosslink of the plasmid DNA. After 30 min treatment of cisplatin, the reporter was used for subsequent experiments. Co-transfection of the reporter with REV3L overexpression vector significantly enhanced the reporter activity by 2.23 -fold. Moreover, the addition of REV3L and REV7L, which was able to form the complete Pol $\zeta$, significantly increased the reporter expression by $>5$-fold.

REV3L knockdown sensitizes $H 1299$ cells to cisplatin. We investigated the effect of REV3L expression on cell sensitivity to cisplatin treatment. As shown in Fig. 5A and C, transfection of pcDNA3.1-REV3L or shRNA-REV3L did not cause an obvious change of cell viability in H1299 cells. The cells were transfected with pcDNA3.1-REV3L, shRNAREV3L or control vectors for $24 \mathrm{~h}$, and then treated with 10 of $25 \mu \mathrm{M}$ cisplatin for another $48 \mathrm{~h}$. The results revealed that a forced expression of REV3L significantly increased cell viability (Fig. 5A and B), while the knockdown of REV3L significantly decreased the viability of H1299 cells treated with cisplatin (Fig. 5C and D), compared with cisplatin treatment alone. These results demonstrated that silencing of REV3L effectively enhanced the anticancer efficacy of cisplatin.
REV3L silencing promotes cisplatin-induced apoptosis. DAPI staining of nucleus was used to observe morphological changes such as nuclear condensation and fragmentation. The results showed that the nuclear condensation and fragmentation of shRNA-REV3L cells were more pronounced than shRNA-NC $\mathrm{H} 1299$ cells after $10 \mu \mathrm{M}$ cisplatin exposure for $48 \mathrm{~h}$ (Fig. 6A).

We investigated whether silencing of REV3L affected cell apoptosis following treatment with cisplatin. As shown in Fig. 6B and C, knockdown of REV3L did not induce apoptosis (Annexin $\mathrm{V}^{+} / \mathrm{PI}^{-}$plus Annexin $\mathrm{V}^{+} / \mathrm{PI}^{+}$cells). However, silencing of REV3L enhanced the response of $\mathrm{H} 1299$ cells to 10 or $25 \mu \mathrm{M}$ cisplatin (shRNA-NC $+10 \mu \mathrm{M}$ cisplatin $12.41 \%$ vs. shRNAREV3L + $10 \mu \mathrm{M}$ cisplatin $25.37 \%, \mathrm{P}<0.05$; (shRNA-NC + $25 \mu \mathrm{M}$ cisplatin $31.84 \%$ vs. shRNA-REV3L $+25 \mu \mathrm{M}$ cisplatin $46.68 \%, \mathrm{P}<0.05)$. Taken together, these results demonstrated that REV3L inhibition enhances apoptotic cell death of H1299 cells in response to cisplatin.

\section{Discussion}

Repair of cisplatin-induced DNA damage has focused on the NER MMR and HR pathways (11). In a study conducted to explore the contribution of DNA damage response pathways 
A

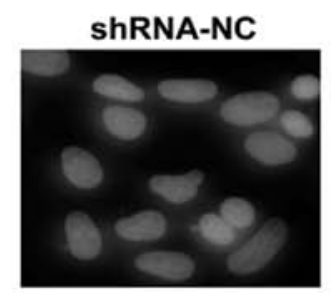
ShRNA-REV3L

B
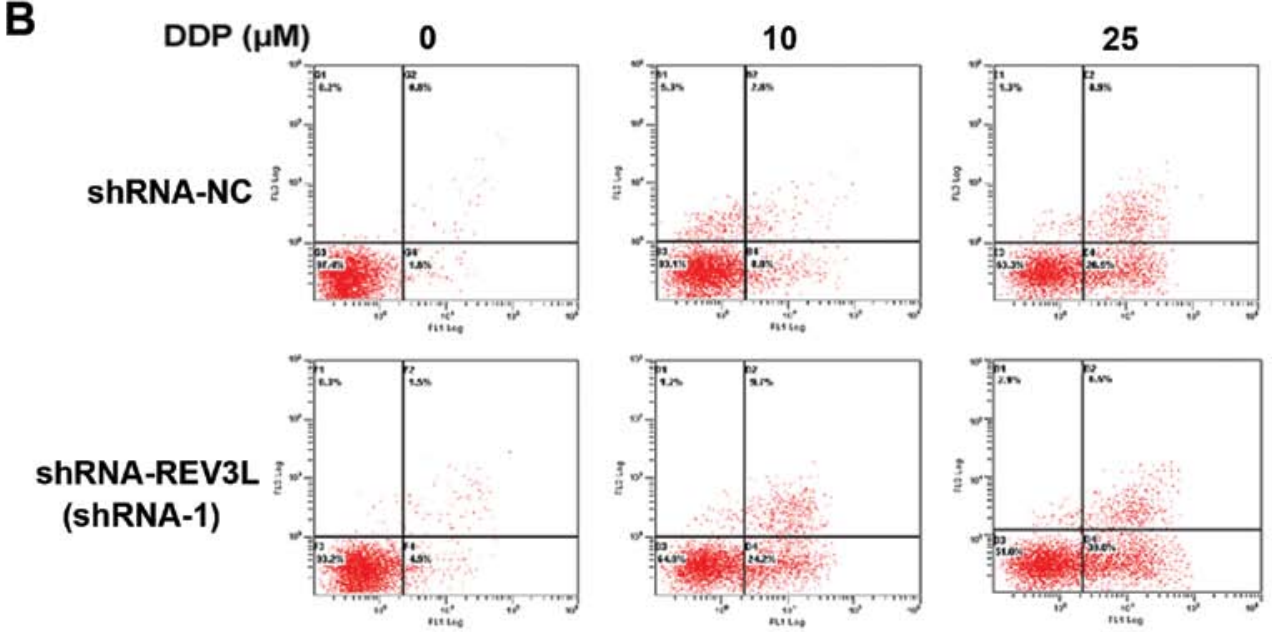

C

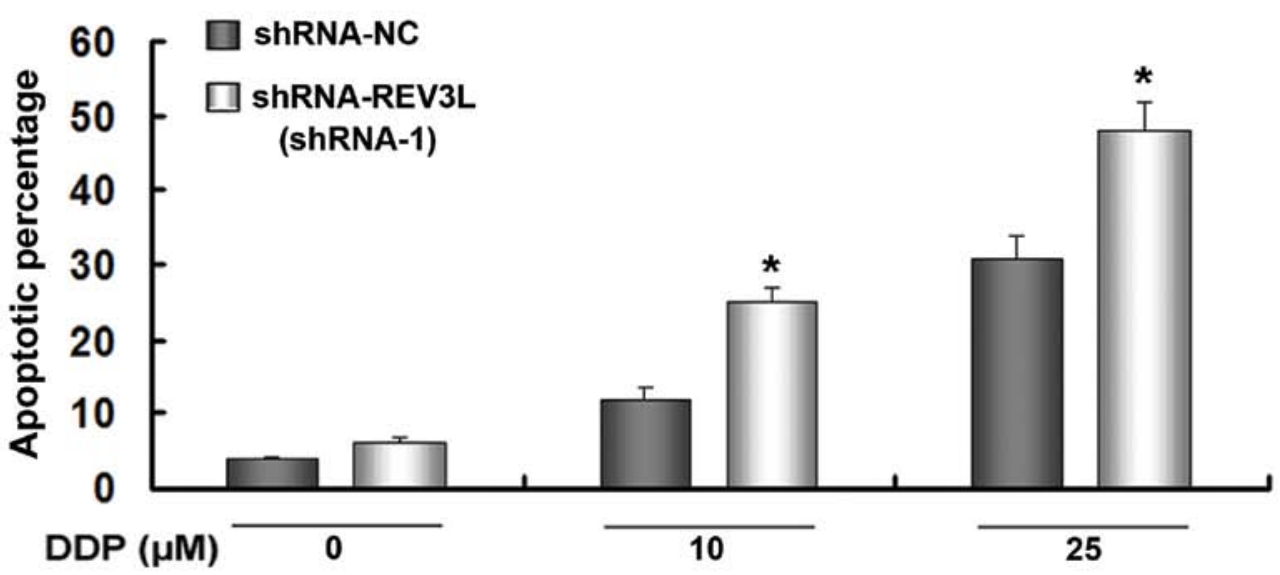

Figure 6. REV3L silencing promotes cisplatin-induced apoptosis. (A) Morphological examination to detect apoptosis by DAPI staining. Nuclear condensation and fragmentation of the REV3L knockdown of H1299 cells were more prominent than the vector control H1299 cells after treatment with $10 \mu$ M cisplatin (DDP) for $48 \mathrm{~h}$. The cells were transfected with the indicated shRNA $24 \mathrm{~h}$ prior to 0,10 or $25 \mu \mathrm{M}$ cisplatin. Cell apoptosis was measured using propidium iodide (PI)/Annexin V double staining in H1299 cells. (B) Representative image of apoptotic analysis. (C) Calculated apoptotic percentage of the group of cells. The data are shown as mean \pm SEM for three independent experiments. Statistical analysis between the groups was determined by ANOVA; ${ }^{*}<0.05$.

in tolerance to cross-linking agents in vertebrates, a panel of gene-disrupted clones from chicken DT40 cells was used to measure the sensitivities of chicken DT40 cells to cross-linking agents, including cisplatin, mitomycin C, and melphalan. It was found that cells harboring defects in the TLS pathway, Fanconi anemia complementation groups (FANC) or homologous recombination pathway exhibited marked hypersensitivity to all the cross-linking agents, whereas NER played only a minor role (24). In particular, cells deficient in REV3 showed the highest sensitivity and markedly increased chrosomal abberrations to cisplatin (24).

The role of low-fidelity TLS polymerases in tumorigenesis remains controversial, because their existence is beneficial for the survival of human cells, while it accumulates mutations during DNA replication (25). Given the importance of TLS participants, studies have attempted to increase the efficacy of chemotherapy by inhibiting this pathway. Albertella et al found that Pol $\eta$ is migrated to nucleus in response to cisplatin and its deficiency significantly decreased cell focus formation after cisplatin treatment (26). DNA polymerase $\eta$ could replicate across intrastrand cross-link between cisplatin and two adjacent $\mathrm{G}$ residues (27). Wu et al found that cisplatin induced a concentration- and time-dependent increase in hREV3 mRNA and suppression of REV3L by transfection of a vector-expressing hREV3 antisense mRNA increased cisplatin sensitivity in human fibroblast (28).

hREV1, another important member in translesional replication and a Pol $\zeta$ interaction molecule, was also upregulated 
in response to cisplatin in human ovarian carcinoma 2008 cells (29). Inactivation of REV1 causes it to become hypersensitive to a wide variety of DNA-damaging agents including cisplatin in human ovarian cancer cells $(29,30)$. Similar results were obtained in colon cancer HCT-116 cells where the introduction of a shRNA against REV3L reduced the mutagenic bypass of cisplatin and cisplatin resistance. Comparatively, inhibition of the DNA MMP pathway did not have a significant effect on reporter expression, which emphasizes the importance of REV3L (17). Suppression of the structual unit of Pol ל-REV7L (MAD2B) conferred hypersensitivity to a range of DNA-damaging agents, especially DNA cross-linkers such as cisplatin and $\gamma$-irradiation (31), suggesting that each of the TLS enzymes may have specificity to the chemotherapeutic drugs. We have previously reported that the downregulation of REV3L sensitized glioma cells to cisplatin via mitochondriamediated apoptosis (32). Taken together, those results indicate that TLS enzymes are activated in response to chemotherapy as endogenous adaptive mechanisms and inhibition of these polymerases contributes to the efficacy of cisplatin.

In this study, we also found that transcription factor $\mathrm{Sp} 1$ is involved in cisplatin-induced REV3L overexpression. In addition, increased Sp1 binding was found in the proximal region of the $R E V 3 L$ promoter. To the best of our knowledge, this is the first study to show the transcriptional control of REV3L following cisplatin exposure. Sp1 is a ubiquitously expressed zinc finger-containing DNA-binding protein that can activate or repress gene transcription in response to various physiologic and pathological stimuli (33). Sp1 binds to GC-rich recognition elements (GC-boxes) through its $\mathrm{C}$-terminal zinc finger motifs $(34,35)$. Sp1 has been reported to be activated in response to oxidative stress and regulate neuronal survival in cortical neurons (36). DNA damage induces transient elevation in its DNA binding activity and phosphorylation of Ser-56 and Ser-101 residues on Sp1 in an ATM-dependent manner $(35,37)$. In cancer cells, $\mathrm{Sp} 1$ is involved in the transcriptional control of pro-apoptotic NOXA following cisplatin exposure (38). Our results identified a new downstream target of $\mathrm{Spl}$ in response to cisplatin.

Taken together, we have demonstrated that cisplatin induced the expression of REV3L by recruiting Sp1 to its promoter. The knockdown of REV3L sensitized cisplatin efficacy in human lung cancer H1299 cells. Similar results were obtained when the ability of the cells to express luciferase from a platinated plasmid was measured.

\section{Acknowledgements}

This study is supported by the National Natural Science Foundation of China (81472920, 81472917, 31400720 and 81372433), Suzhou Administration of Science and Technology (SYS201416) and the Priority Academic Program Development of Jiangsu Higher Education Institutions (PAPD).

\section{References}

1. Jemal A, Siegel R, Xu J and Ward E: Cancer statistics, 2010. CA Cancer J Clin 60: 277-300, 2010.

2. Langer CJ, Mok T and Postmus PE: Targeted agents in the third-/ fourth-line treatment of patients with advanced (stage III/IV) non-small cell lung cancer (NSCLC). Cancer Treat Rev 39 252-260, 2013
3. de Boer RH, Arrieta O, Yang CH, Gottfried M, Chan V, Raats J, de Marinis F, Abratt RP, Wolf J, Blackhall FH, et al: Vandetanib plus pemetrexed for the second-line treatment of advanced nonsmall-cell lung cancer: A randomized, double-blind phase III trial. J Clin Oncol 29: 1067-1074, 2011.

4. O'Rourke N, Roqué I Figuls M, Farré Bernadó N and Macbeth F: Concurrent chemoradiotherapy in non-small cell lung cancer. Cochrane Database Syst Rev 6: CD002140, 2010.

5. Barr MP, Gray SG, Hoffmann AC, Hilger RA, Thomale J, O'Flaherty JD, Fennell DA, Richard D, O'Leary JJ and O'Byrne KJ: Generation and characterisation of cisplatinresistant non-small cell lung cancer cell lines displaying a stem-like signature. PLoS One 8: e54193, 2013.

6. Einhorn LH: First-line chemotherapy for non-small-cell lung cancer: Is there a superior regimen based on histology? J Clin Oncol 26: 3485-3486, 2008.

7. Rossi A, Di Maio M, Chiodini P, Rudd RM, Okamoto H, Skarlos DV, Früh M, Qian W, Tamura T, Samantas E, et al: Carboplatin- or cisplatin-based chemotherapy in first-line treatment of small-cell lung cancer: The COCIS meta-analysis of individual patient data. J Clin Oncol 30: 1692-1698, 2012.

8. Galluzzi L, Senovilla L, Vitale I, Michels J, Martins I, Kepp O, Castedo M and Kroemer G: Molecular mechanisms of cisplatin resistance. Oncogene 31: 1869-1883, 2012.

9. Wang D and Lippard SJ: Cellular processing of platinum anticancer drugs. Nat Rev Drug Discov 4: 307-320, 2005.

10. Martin LP, Hamilton TC and Schilder RJ: Platinum resistance: The role of DNA repair pathways. Clin Cancer Res 14: 1291-1295, 2008.

11. Basu A and Krishnamurthy S: Cellular responses to Cisplatininduced DNA damage. J Nucleic Acids 2010: 201367, 2010.

12. Kelland L: The resurgence of platinum-based cancer chemotherapy. Nat Rev Cancer 7: 573-584, 2007.

13. Friedberg EC, Lehmann AR and Fuchs RP: Trading places: How do DNA polymerases switch during translesion DNA synthesis? Mol Cell 18: 499-505, 2005.

14. Gan GN, Wittschieben JP, Wittschieben BØ and Wood RD: DNA polymerase zeta (pol zeta) in higher eukaryotes. Cell Res 18: 174-183, 2008.

15. Okada T, Sonoda E, Yoshimura M, Kawano Y, Saya H, Kohzaki M and Takeda S: Multiple roles of vertebrate REV genes in DNA repair and recombination. Mol Cell Biol 25: 6103-6111, 2005.

16. Shen X, Jun S, O'Neal LE, Sonoda E, Bemark M, Sale JE and Li L: REV3 and REV1 play major roles in recombinationindependent repair of DNA interstrand cross-links mediated by monoubiquitinated proliferating cell nuclear antigen (PCNA). J Biol Chem 281: 13869-13872, 2006.

17. Wittschieben JP, Reshmi SC, Gollin SM and Wood RD: Loss of DNA polymerase zeta causes chromosomal instability in mammalian cells. Cancer Res 66: 134-142, 2006.

18. Pan Q, Fang Y, Xu Y, Zhang K and Hu X: Down-regulation of DNA polymerases kappa, eta, iota, and zeta in human lung, stomach, and colorectal cancers. Cancer Lett 217: 139-147, 2005.

19. Zhang S, Chen H, Zhao X, Cao J, Tong J, Lu J, Wu W, Shen H, Wei Q and Lu D: REV3L 3'UTR $460 \mathrm{~T}>\mathrm{C}$ polymorphism in microRNA target sites contributes to lung cancer susceptibility. Oncogene 32: 242-250, 2013.

20. Lin X, Trang J, Okuda T and Howell SB: DNA polymerase zeta accounts for the reduced cytotoxicity and enhanced mutagenicity of cisplatin in human colon carcinoma cells that have lost DNA mismatch repair. Clin Cancer Res 12: 563-568, 2006.

21. Eastman A, Jennerwein MM and Nagel DL: Characterization of bifunctional adducts produced in DNA by transdiamminedichloroplatinum(II). Chem Biol Interact 67: 71-80, 1988.

22. Zhang S, Lu J, Zhao X, Wu W, Wang H, Lu J, Wu Q, Chen X, Fan W, Chen H, et al: A variant in the CHEK2 promoter at a methylation site relieves transcriptional repression and confers reduced risk of lung cancer. Carcinogenesis 31: 1251-1258, 2010.

23. Jamieson ER and Lippard SJ: Structure, recognition, and processing of cisplatin-DNA adducts. Chem Rev 99: 2467-2498, 1999.

24. Nojima K, Hochegger H, Saberi A, Fukushima T, Kikuchi K, Yoshimura M, Orelli BJ, Bishop DK, Hirano S, Ohzeki M, et al: Multiple repair pathways mediate tolerance to chemotherapeutic cross-linking agents in vertebrate cells. Cancer Res 65: 11704-11711, 2005.

25. Lange SS, Takata K and Wood RD: DNA polymerases and cancer. Nat Rev Cancer 11: 96-110, 2011.

26. Albertella MR, Green CM, Lehmann AR and O'Connor MJ: A role for polymerase eta in the cellular tolerance to cisplatininduced damage. Cancer Res 65: 9799-9806, 2005. 
27. Alt A, Lammens K, Chiocchini C, Lammens A, Pieck JC, Kuch D, Hopfner KP and Carell T: Bypass of DNA lesions generated during anticancer treatment with cisplatin by DNA polymerase eta. Science 318: 967-970, 2007.

28. Wu F, Lin X, Okuda T and Howell SB: DNA polymerase zeta regulates cisplatin cytotoxicity, mutagenicity, and the rate of development of cisplatin resistance. Cancer Res 64: 8029-8035, 2004.

29. Okuda T, Lin X, Trang J and Howell SB: Suppression of hREV1 expression reduces the rate at which human ovarian carcinoma cells acquire resistance to cisplatin. Mol Pharmacol 67: 1852-1860, 2005.

30. Lin X, Okuda T, Trang J and Howell SB: Human REV1 modulates the cytotoxicity and mutagenicity of cisplatin in human ovarian carcinoma cells. Mol Pharmacol 69: 1748-1754, 2006.

31. Cheung HW, Chun AC, Wang Q, Deng W, Hu L, Guan XY, Nicholls JM, Ling MT, Chuan Wong Y, Tsao SW, et al: Inactivation of human MAD2B in nasopharyngeal carcinoma cells leads to chemosensitization to DNA-damaging agents. Cancer Res 66: 4357-4367, 2006.

32. Wang H, Zhang SY, Wang S, Lu J, Wu W, Weng L, Chen D, Zhang Y, Lu Z, Yang J, et al: REV3L confers chemoresistance to cisplatin in human gliomas: The potential of its RNAi for synergistic therapy. Neuro-oncol 11: 790-802, 2009.
33. Tan NY and Khachigian LM: Sp1 phosphorylation and its regulation of gene transcription. Mol Cell Biol 29: 2483-2488, 2009.

34. Fojas de Borja P, Collins NK, Du P, Azizkhan-Clifford J and Mudryj M: Cyclin A-CDK phosphorylates Sp1 and enhances Sp1-mediated transcription. EMBO J 20: 5737-5747, 2001.

35. Iwahori S, Yasui Y, Kudoh A, Sato Y, Nakayama S, Murata T, Isomura $\mathrm{H}$ and Tsurumi T: Identification of phosphorylation sites on transcription factor $\mathrm{Sp} 1$ in response to DNA damage and its accumulation at damaged sites. Cell Signal 20: 1795-1803, 2008.

36. Ryu H, Lee J, Zaman K, Kubilis J, Ferrante RJ, Ross BD, Neve R and Ratan RR: Sp1 and Sp3 are oxidative stress-inducible, antideath transcription factors in cortical neurons. J Neurosci 23 : 3597-3606, 2003.

37. Meighan-Mantha RL, Riegel AT, Suy S, Harris V, Wang FH, Lozano $\mathrm{C}$, Whiteside TL and Kasid U: Ionizing radiation stimulates octamer factor DNA binding activity in human carcinoma cells. Mol Cell Biochem 199: 209-215, 1999.

38. Grande L, Bretones G, Rosa-Garrido M, Garrido-Martin EM, Hernandez T, Fraile S, Botella L, de Alava E, Vidal A, Garcia del Muro X, et al: Transcription factors Sp1 and p73 control the expression of the proapoptotic protein NOXA in the response of testicular embryonal carcinoma cells to cisplatin. J Biol Chem 287: 26495-26505, 2012. 